\section{A hidden GEMM}

The second messenger cyclic dimeric guanosine monophosphate (c-di-GMP) induces wide-ranging physiological changes in diverse bacterial species, but how it controls the transcription and translation of so many genes has been unclear. Now, reporting in Science, Sudarsan, Breaker and colleagues describe how, in many bacterial species, c-di-GMP is sensed by riboswitches.

The number of known protein interactions that involve c-di-GMP cannot explain its global cellular

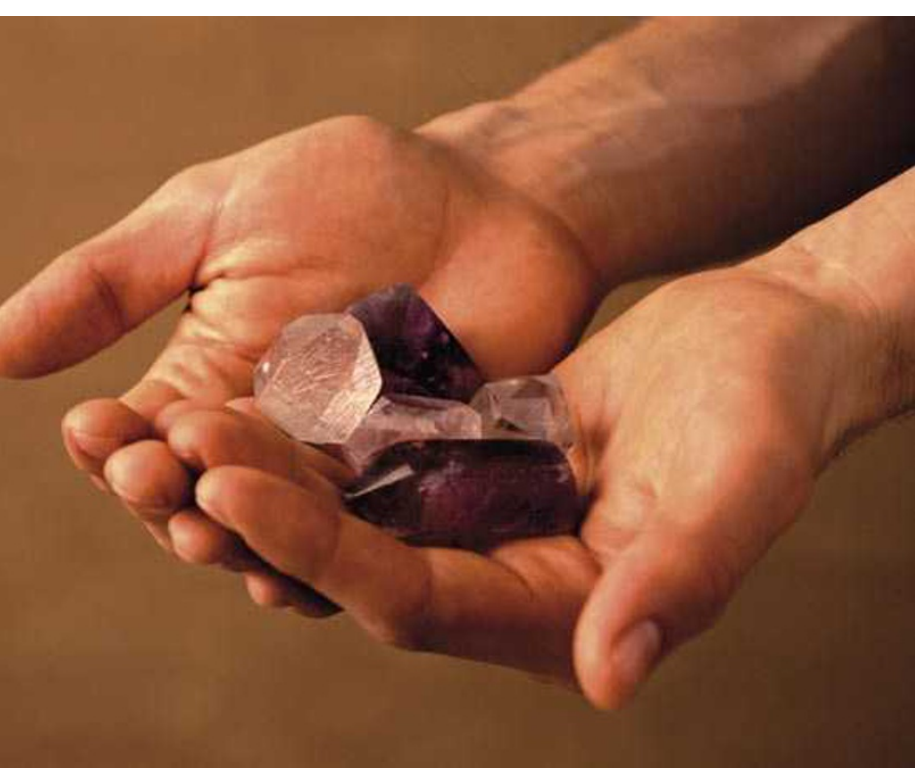

effects, but it has been proposed that these effects could be explained by the existence of c-di-GMP riboswitches. Riboswitches are complex, structured RNA domains that function as receptors for specific metabolites. They are found in the non-coding regions of various mRNAs and control gene expression in response to metabolite binding.

Sudarsan et al. discovered that the upstream regions of several genes that are controlled by c-di-GMP contain a highly conserved RNA domain called GEMM (genes for the environment, for membranes and for motility). They subjected two GEMM motifs from Vibrio cholerae (Vc1 and $\mathrm{Vc} 2$ ) to an RNA-structure probing method in the absence and presence of c-di-GMP. This method identified nucleotides in these GEMM motifs that are important for c-di-GMP binding and showed that they bind c-di-GMP tightly and specifically.

Next, the authors investigated whether GEMM motifs from $V$. cholerae (Vc2), Bacillus cereus (Bc1 and $\mathrm{Bc} 2)$ and Clostridium difficile (Cd1) could function as riboswitches in cells. They transformed cells using a construct that was composed of the 5 -untranslated region in which each GEMM motif was fused to a reporter gene. The construct that carried Vc2 showed high levels of reporter-gene expression, and mutations that disrupted the c-di-GMP-binding properties of $\mathrm{Vc} 2$ reduced these levels. This indicates that Vc2 has an 'on'-switch function, which was also the case for Bc1, whereas an 'off'switch function was identified for $\mathrm{Bc} 2$ and $\mathrm{Cd} 1$.

Modulation of gene expression by a c-di-GMP riboswitch was established by further analysing the off-switch function of Cd1. Sudarsan et al. used a c-di-GMP-degrading enzyme to manipulate the cellular concentration of c-di-GMP and showed that, in the presence of this enzyme, reporter-gene expression that was under the control of $\mathrm{Cd} 1$ was switched on.

Sudarsan et al. have therefore shown that c-di-GMP is sensed by riboswitches in eubacteria, which explains how it controls the expression of genes that are involved in numerous fundamental cellular processes from bacterial virulence to flagellum biosynthesis.

Rachel Smallridge

ORIGINAL RESEARCH PAPER Sudarsan, N. et al. Riboswitches in eubacteria sense the second messenger cyclic di-GMP. Science 321 411-413 (2008)

FURTHER READING Winkler, W. C. \& Breaker, R. R. Regulation of bacterial gene expression by riboswitches. Ann. Rev. Microbiol. 59, 487-517 (2005) 